\title{
ACERCA DE LA DESAPARICIÓN DEL ANTEFuturo DE SUBJUNTIVO DEL ESPAÑOL PENINSULAR
}

\author{
WiTOLD SOBCZAK \\ Universidad de Łódź
}

Title: On the disappearance of the future perfect subjunctive from Peninsular Spanish

Abstract: The aim of this article is to comment on the elimination of the future perfect subjunctive (bubiere cantado) from Peninsular Spanish. Apart from drawing attention to the history, the usage and the causes of the disappearance of this form, the author uses the data gathered in the Corpus Diachronic of Spanish (CORDE) to register the first symptoms of its weakness in the Spanish verb system.

Key words: Future subjunctive. Future perfect subjunctive. Posteriority. Compound tenses. Verb tenses.

\section{INTRODUCCIÓN}

No cabe la menor duda de que el antefuturo de subjuntivo (bubiere cantado) es una forma poco estudiada en comparación con el futuro de subjuntivo (cantare), al que se ha dedicado mucha atención, analizando, entre otras cuestiones, las causas de su desaparición. El hecho de que la forma compuesta despierte menor interés que la simple puede estar vinculado con su empleo relativamente reducido ya en la Edad Media y en los siglos posteriores (Ramos y Folgar, 1992: 408), lo cual se debe al valor temporal denotado por dicho tiempo. Recordemos que hubiere cantado expresaba una acción contingente anterior a otra que era posterior a algún punto de referencia (NGLE, 2009: 1812-1813), es decir, su significado correspondía a situaciones poco frecuentes en la vida cotidiana. Además, a nuestro juicio, la escasez de estudios sobre este tiempo se ve relacionada con cierta tendencia a entender el futuro de subjuntivo y su correspondiente compuesto como un solo tiempo con dos formas, cuestión en la que se fijó en su día Zamorano Aguilar (2005: 360). Observemos que ambos tiempos no tenían equivalentes en latín clásico (Penny, 2014: 194), se sometían desde siempre a numerosas restricciones sintácticas y actualmente su empleo está limitado a los textos 
jurídicos, administrativos, etc. En otras palabras, cantare y bubiere cantado tenían mucho en común y "la diferencia entre ellas era solo temporal, postura que resulta, cuando menos, discutible" (Zamorano Aguilar, 2005: 361).

Dado el escaso número de trabajos acerca del empleo del antefuturo de subjuntivo, en las líneas que siguen intentaremos presentar concisa y brevemente su historia, centrando la atención en las causas de su eliminación del español peninsular. Para determinar en qué siglo empieza a caer en desuso la forma que nos interesa, aprovecharemos los datos recopilados en el corpus CORDE.

\section{Origen y uso del antefuturo de subjuntivo}

Como señala Penny (2014: 194), el tiempo antefuturo de subjuntivo carecía de antecedentes latinos y se creó como equivalente del paradigma del futuro de subjuntivo (Habere Habuero/Habuerim CANTATUM) $>$ ovier(e) cantado > bubiere cantado, etc. Sin lugar a dudas, la forma compuesta (bubiere cantado) se documenta más tarde que la simple (cantare), siendo rarísima en los primeros documentos literarios, al igual que los demás tiempos compuestos del subjuntivo cuyo desarrollo se registra hacia mediados del siglo XIII ${ }^{1}$ (Andres Suárez, 1994: 277).

Desde el punto de vista sintáctico, el antefuturo de subjuntivo era empleado ante todo en oraciones relativas con pronombre que y con antecedente expreso determinado. El determinante aparecía como primer elemento de una frase nominal o precedía directamente a la oración relativa:

1. [...] conuiene que tales obras fagan por que con derecho acresçienten su regno et fagan en guisa que en su vida sean muy loados de las gentes, et despues de su muerte

${ }^{1}$ Curiosamente en la misma época, es decir, a mediados del siglo XIII la forma cantare demuestra los primeros indicios de debilidad, ya que su empleo empieza a retroceder, aunque todavía no de forma significativa ante una frecuencia cada vez más elevada de las formas sustitutas (Luquet, 1988: 168; Camus Bergareche, 1990: 420; Andres Suárez, 1994: 159; De Sterck, 2000: 267). 
finquen buenas fazannas de las buenas obras que ellos ovieren fechas. (Lucanor, 41, 30, apud Folgar y Ramos, 1992: 415)

2. [...] a las que fueren costunbradas de comer dos uezes al dja çéuenlas a la ora de las biésperas. Cetrería, 14, 8, apud Folgar y Ramos, 1992: 415

Amén de ello, bubiere cantado se utilizaba con frecuencia en oraciones subordinadas adverbiales introducidas por algunas de las siguientes conjunciones o locuciones conjuntivas: como, después que, desque, pues que, quando y si:

3. [...] S. M. desea tanto venir á proseguir las que aquí dejó comenzadas, que no dará lugar á mas tiempo, sino á concedellos algunas gracias, como seria quitarles los presidios del reino, y perdonar algunos caballeros y personas que estan culpadas de las inquietudes pasadas; esto se irá entendiendo como hubiere entrado en el reino. (Relación de las cosas sucedidas en la corte de España desde 1599 hasta 1614, Cabrera de Córdoba Luis, c 1599-1614 en CORDE)

4. [...] las sesenta y dos libras y media antes de principiar la obra, y quando hubiere acabado el quadro de medio del Baptismo de Xesucristo Nuestro Redentor... (Alberto Claramun, señor de Artajona, encarga al pintor Pedro Orfelín nueve lienzos para su capilla e ..., Anónimo, 1605 en CORDE)

Asimismo, el antefuturo de subjuntivo denotaba una situación contingente anterior a otra que era posterior a algún punto de referencia (NGLE, 2009: 1812-1813):

5. Si dentro de treinta días a partir de la fecha de inscripción del registro del jugador, los clubes no hubieren llegado a un acuerdo sobre el monto de la indemnización, el Comité Ejecutivo tiene facultad para establecer el monto de la indemnización. (ABC Color 17/9/1996, apud NGLE, 2009: 1814) 
Está claro que la prótasis condicional aporta en (5) el contexto modal (Si dentro de treinta días...). El texto informa, por tanto, de que algo ocurrirá si la consecución de cierto acuerdo no tiene lugar en un momento anterior al final de un lapso de treinta días que se cuentan desde el momento en que se realiza determinada inscripción.

Hoy en día, la forma compuesta del futuro de subjuntivo sigue apareciendo en los textos jurídicos con el fin de atenuar el carácter hipotético de la situación que se describe. Sin embargo, desde hace tiempo viene reemplazada en muchas ocasiones por bubiera-se cantado, tanto en el sentido en el que hace referencia a lo venidero como en el que alude a una situación pasada que se considera improbable:

6. En el caso de que se hubieren producido esas circunstancias, entonces el proyecto histórico que se propuso escribir, habría sido notoriamente acertado. (Magallón, Renacimiento, apud NGLE, 2009: 1815)

Por último, el antefuturo de subjuntivo puede expresar probabilidad con respecto al presente o antepresente. De este modo, hubiere venido admite la paráfrasis puede (ser) que haya venido en el texto siguiente:

7. Yo creo que la vida no debe ser una meditación sobre la muerte sino una meditación sobre la vida, y solo me retiene a su lado la gente que mira las cosas, aun las más insignificantes, aun las que parecen invitar menos a la meditación, con la actitud de quien hubiere venido desde una región noble a este planeta para hacer una compulsa sincera. (Mallea, Bahía, apud NGLE, 2009: 1815)

Conviene subrayar que no existe unanimidad entre los estudiosos sobre los tiempos que sustituyeron al antefuturo de subjuntivo, lo que nos recuerda la polémica entre Rojo y Montero, y Veiga en torno a las formas que reemplazaron al futuro de subjuntivo en las oraciones condicionales. Rojo y Montero (1983) afirmaban que "si tuviere" se reemplazó con "si tuviese" o 
"si tuviera”, opinión con la que polemizaba Veiga (1989), destacando que "si tuviere" cedió lugar a "si tengo". Ese problema también atrajo la atención de Nowikow (1995: 114) quien consideraba indispensable distinguir entre el «cambio nuclear» y el «cambio periférico» (acompañante). El primero se referiría a la sustitución de cantare por cante o canta, mientras que el segundo se produciría mediante la "invasión" de cantara en las «zonas» reservadas en principio para el futuro de subjuntivo ${ }^{2}$.

En lo referente al reemplazo del antefuturo de subjuntivo, Gili Gaya (1994 [1943]: 183) advertía que las funciones de bubiere cantado las han tomado haya cantado y hubiera-se cantado. Sin embargo, a la vista del valor temporal del antefuturo de subjuntivo que acabamos de ver supra, nos convence más la opinión de Lope Blanch (1972: 141-142), según la cual las funciones de dicho tiempo se reparten en el español contemporáneo entre el antecopretérito de subjuntivo (bubiera-se cantado), el antepresente de subjuntivo (baya cantado) y el presente de subjuntivo (cante):

8. Si alguien infringe esta disposición, será castigado con arreglo al daño que haga. (Lope Blanch, 1972: 142)

Considerar el presente de subjuntivo como uno de los sustitutos del antefuturo de subjuntivo parece perfectamente comprensible si tenemos en cuenta que cante reemplaza en algunas ocasiones al contenido temporal transmitido por baya cantado ${ }^{3}$ :

9. Cuando lo leas/haya leído, me lo devuelves.

\footnotetext{
${ }^{2}$ Pare leer más sobre esta cuestión remitimos al lector al artículo de Nowikow (1995).

${ }^{3}$ Esto no quiere decir que el antepresente de subjuntivo desaparezca de la lengua contemporánea. En realidad, es un tiempo verbal que goza de vigencia y que, además de cumplir la función que le corresponde, sustituye con frecuencia al antecopretérito de subjuntivo (bubiera cantado) en gran parte de Sudamérica. Para leer más sobre este fenómeno véase Veiga (2011).
} 
10. En cuanto se duerma/se haya dormido, nos vamos.

(Lope Blanch, 1972: 147))

El caso aún más peculiar lo constituye el uso del antefuturo de subjuntivo en la prótasis de oraciones condicionales en la que el tiempo en cuestión es sustituido por el presente o el antepresente de indicativo:

11. Si dentro de treinta días a partir de la fecha de inscripción del registro del jugador, los clubes no hubieren llegado/llegan/han llegado a un acuerdo sobre el monto de la indemnización, el Comité Ejecutivo tiene facultad para establecer el monto de la indemnización. (ABC Color 17/9/1996, apud NGLE, 2009: 1814)

Como se ve, en (11) podríamos emplear llegan o han llegado en vez bubieren llegado para denotar una situación contingente anterior a otra que es posterior al origen.

Vista la complejidad de las relaciones temporales en el sistema temporal castellano, creemos que las formas que desempeñaron un papel decisivo en la eliminación de bubiere cantado ${ }^{4}$ del sistema temporal castellano fueron haya cantado y cante, mientras que en la prótasis de oraciones condicionales la forma que nos ocupa quedó reemplazada por canta y ha cantado a causa de una serie de restricciones de índole sintáctica. En cambio, la sustitución de bubiere cantado por hubiera-se cantado puede considerarse un cambio acompañante y se parece a la mencionada «invasión» de cantara en las zonas ocupadas originalmente por cantare. El hecho de que el futuro de subjuntivo y su correlato compuesto fueran sustituidos por más de un tiempo verbal parece aún más comprensible si tenemos en cuenta que el grado de exactitud de localización temporal $\left(\mathrm{GELT}^{5}\right)$ de los tiempos del subjuntivo es

\footnotetext{
${ }^{4}$ Andres Suárez (1994: 268) destaca que "todos los tiempos del subjuntivo son aptos para expresar acción futura; por consiguiente, han ido haciéndose innecesarios tanto el futuro simple como el compuesto de dicho modo [...]". En nuestra opinión, tal afirmación requiere indudablemente algunas precisiones, sobre todo con respecto a las formas compuestas que en principio denotan anterioridad.

${ }^{5}$ Criterio introducido y descrito detalladamente por Nowikow (1993) y (2010).
} 
inferior a los del indicativo. A nuestro modo de ver, esta diferencia se debe, entre otros, al número de tiempos de los que disponen dichos modos ( 4 vs. 9!). A consecuencia de ello, es natural que tanto cantare como bubiere cantado se reemplazaran por más de un tiempo verbal para "rellenar los huecos" temporales y modales dejados por ellas en el sistema temporal castellano.

3. Desaparición del antefuturo de subjuntivo Del sistema TEMPORAL CASTELLANO

Aunque escasean los trabajos en los que se comenta la eliminación del antefuturo de subjuntivo del sistema temporal castellano, vale la pena subrayar que ya en 1881 Commerelán lo clasifica como un tiempo poco usado, mientras que Lenz en 1920 pone de manifiesto que es un tiempo totalmente anticuado, desaparecido del lenguaje corriente. Seco (1990 [1930]), por su parte, apunta que el antefuturo de subjuntivo tiene cierta funcionalidad hasta el siglo XVIII, limitada sobre todo a las oraciones condicionales, lo que coincide en gran medida con las observaciones de Penny (2014: 247), según quien el antefuturo de subjuntivo desaparece a partir del siglo XVIII. Curiosamente, en la datación propuesta por estos lingüistas no se distingue entre el español peninsular y su variedad americana, factor que puede ser relevante a la luz de la polémica acerca de la desaparición de la forma simple (cantare) en el español de América y en las Islas Canarias ${ }^{6}$.

A la hora de tratar la eliminación de bubiere cantado, hay que tener presente que estamos ante un tiempo compuesto, es decir, se trata de

una creación románica basada en la desemantización del verbo auxiliar y en la gramaticalización paulatina de la construcción que desempeña un papel importante en el establecimiento de las relaciones de anterioridad siempre

\footnotetext{
${ }^{6}$ Según algunos estudiosos cantare no solo pervivía todavía en el siglo XX en el norte de Colombia, Puerto Rico, Santo Domingo (Granda, 1968; Álvarez, 1972; Jorge Morel, 1974), sino que también se documentaba en las Islas Canarias (Donni de Mirande, 1984; Luquet, 1988). Por lo que se refiere al continente americano, hemos de tener en cuenta que la afirmación sobre la pervivencia del futuro de subjuntivo no ha sido corroborada por muchos otros estudiosos (Veiga, 2006: 136).
} 
con respecto al tiempo de su verbo auxiliar: ha escrito anterior a escribe, habrá escrito anterior a escribirá (Nowikow, 2012: 132).

Sin distinguir entre los modos indicativo y subjuntivo, parece que los tiempos compuestos resultan por varios motivos "menos resistentes" frente a los simples a los cambios sucedidos en el sistema verbal del español a partir de la Edad Media (en el castellano contemporáneo no se emplean prácticamente las formas bubo cantado y bubiere cantado). En cambio, el único tiempo simple que dejó de usarse durante el pasado del español medieval al español actual fue el futuro de subjuntivo. De este modo, el número de tiempos se ha reducido a 7 formas compuestas (incluidas en estas los alomorfos en -se y -ra) y a 8 simples (también incluidas en estas los alomorfos en -se y en -ra). Fijémonos en que en el otoño de la Edad Media y en los Siglos de Oro, es decir, en la época en la que se produjo una consolidación de las formas compuestas, el número de tiempos simples y compuestos era igual en ambos modos (cinco tiempos en el modo indicativo y cuatro tiempos en el subjuntivo).

\section{ANÁLISIS DEL CORPUS}

Tal y como hemos señalado supra, en nuestro análisis del empleo del antefuturo de subjuntivo manejamos los datos recopilados en el corpus CORDE con la finalidad de precisar el momento en el que dicha forma empieza a perder vigor en el español peninsular. Los ejemplos estudiados (1180) com-

\footnotetext{
${ }^{7}$ En este caso no nos referimos exclusivamente a los tiempos compuestos que han desaparecido del sistema temporal castellano, sino que también consideramos oportuno llamar la atención sobre tales tendencias como el reemplazo de ha cantado por cantó en gran parte del mundo hispanohablante y el uso actual de habrá cantado que queda limitado al valor modal (su valor temporal tiende a ser sustituido, entre otros, por tener + participio o ir a + infinitivo (Moreno de Alba 2010 [1988]: 239). Sin embargo, todo ello no quiere decir que los tiempos compuestos dejen de utilizarse y no se produzcan cambios entre los tiempos simples. Recordemos que existen múltiples trabajos en los que se estudia pormenorizadamente la sustitución del futuro (cantará) por la perífrasis verbal (va a cantar), es decir, un fenómeno opuesto en el que la forma compuesta reemplaza a la simple.
} 
prenden los siglos $\mathrm{XVI-XX^{8 }}$ y proceden de todos los tipos de textos, salvo la prosa jurídica (documentos notariales, ordenamientos, códigos legales, etc.). Es de subrayar que el lenguaje legal es un caso peculiar en el que se siguen utilizando las formas simple y compuesta del futuro de subjuntivo y, por eso, nos vemos obligados a excluirlo del análisis. Además, hemos decidido eliminar una serie de ejemplos provenientes de la Reina Valera 1909, puesto que es una revisión de la llamada "Biblia del oso" editada en el año 1569, época en la que se empleaba mucho más el antefuturo de subjuntivo que a principios del siglo XX. De esta manera creemos que será más fácil observar el proceso de la paulatina desaparición de bubiere cantado del sistema temporal castellano.

\begin{tabular}{|l|l|}
\hline Forma & Número de ejemplos \\
\hline bubiere cantado & 780 \\
\hline bubieres cantado & 39 \\
\hline bubiéremos cantado & 14 \\
\hline bubiereis cantado & 10 \\
\hline bubieren cantado & 337 \\
\hline Total & $\mathbf{1 1 8 0}$ \\
\hline
\end{tabular}

Tabla 1: Repartición general de las formas del antefuturo de subjuntivo (Fuente: elaboración propia)

Según se aprecia en la tabla 2, la desaparición del antefuturo de subjuntivo en la península ibérica se nota en la lengua escrita a partir del siglo XVIII, aunque sus primeros síntomas se registran en el siglo XVII. Esta última observación no se basa exclusivamente en la cantidad de ejemplos documentados en esas dos centurias, sino que también viene respaldada por la diferencia que se da en la frecuencia de empleo de bubiere cantado en la

${ }^{8}$ En el corpus CORDE hay poquísimos ejemplos procedentes de las centurias anteriores. A pesar de ello, creemos que la época estudiada es bastante extensa y permite observar la decadencia del uso de hubiere cantado.

${ }^{9}$ Debido al número de ejemplos estudiados no distinguimos entre la primera y la tercera persona del singular. La forma hubieres cantado, etc. se usa prototípicamente para referirse a todas las formas de la segunda persona del singular recopiladas en el corpus. 


\begin{tabular}{|l|l|}
\hline Siglo & Número de ejemplos \\
\hline XVI & 439 \\
\hline XVII & 387 \\
\hline XVIII & 98 \\
\hline XIX & 168 \\
\hline XX & 88 \\
\hline Total & 1180 \\
\hline
\end{tabular}

Tabla 2: Repartición de las formas del antefuturo de subjuntivo por siglos (Fuente: elaboración propia)

primera y en la segunda mitad del siglo XVII (345 vs. 42 casos). Todo ello nos hace suponer que el antefuturo de subjuntivo presenta debilidad ya hacia mediados del siglo XVII. El empleo del tiempo en cuestión crece un poco en el siglo XIX, pero no se acerca de ningún modo a la frecuencia con la que se usaba en los siglos XVI y XVII. El siglo XX, a su vez, confirma de nuevo que bubiere cantado es una forma empleada en raras ocasiones.

Aunque el CORDE no nos ofrece la posibilidad de calcular con precisión la frecuencia normalizada del empleo del antefuturo de subjuntivo, hemos de tener presente que los datos recopilados en el corpus están distribuidos de la manera siguiente: Edad Media (1250-1492): $21 \%$; Siglos de Oro (1493-1713): 28 \% y Época Contemporánea (1714-1974): $51 \%$, lo cual, sin lugar a dudas, facilita la interpretación de los resultados obtenidos. A nuestro juicio, el decreciente número de formas del antefuturo de subjuntivo, que se registra con claridad en el siglo XVIII, es inversamente proporcional al número de textos recopilados en el CORDE, cuya cantidad es mucho más representativa para los siglos XVIII-XX (en concreto para el periodo que abarca los años 1714-1974) que para las centurias anteriores. Ese último factor contribuye a que en el siglo XIX se registren más ejemplos de bubiere cantado que en el XVIII y, a consecuencia de ello, puede darse la falsa impresión de que el uso de dicho tiempo verbal era más elevado en el siglo XIX que en el precedente. Además, a tenor de los datos extraídos del corpus, se observa que el antefuturo de subjuntivo, siendo la forma usada sobre todo 
en el registro formal, aparecía raras veces en la segunda persona del singular y en la primera y en la segunda persona del plural:

12. Y si no lo hubieres llamado con fiador, echarle has allí la lonja, atándola en el siñuelo antes que acabe de comer; porque, después de haber comido, no se te levante y se vaya. (Libro de cetrería de caza de azor, 1565, Zúñiga y Sotomayor Fadrique de en CORDE)

13. Yo muy contento, pues pagarse tiene; que, en el cambio de la otra vida, prestan dineros para que paguemos todos los mendigos lo que hubiéremos usurpado a pesar de sus dueños. (El guitón Onofre, 1604, González Gregorio en CORDE)

14. Últimamente ha llegado aquí una carta vuestra fechada el 22 de agosto, por avión; y a los pocos días otra del 20 por un vapor. Me sorprende que el día 22 no hubiereis recibido la primera mía, del 8 de agosto, que mandé por el aire; será debido a vuestros traslados. (Carta [Cartas de Manuel Azaña y Cipriano de Rivas Cherif], 1929, Azaña Manuel en CORDE)

Si aceptamos la afirmación de Andres Suárez de que el desarrollo del antefuturo de subjuntivo se nota a partir de la mitad del siglo XIII, podemos llegar a la conclusión de que su uso "relativamente" frecuente dura unos 400 años. Dicho de otra manera, bubiere cantado es la forma más "débil” de las que formaban originalmente el subsistema de tiempos compuestos al lado de bubo cantado ${ }^{10}$.

\section{A MANERA DE CONCLUSiÓN}

Recapitulando todo lo que hemos dicho, podemos extraer las siguientes conclusiones con respecto al antefuturo de subjuntivo:

1. El tiempo en cuestión empieza a caer en desuso en la península ibérica en el siglo XVIII, pero los primeros síntomas de su debilidad en la

\footnotetext{
${ }^{10}$ Según advierten Octavio de Toledo y Huerta y Rodríguez Molina (2008: 277), el llamado pretérito anterior (bubo cantado) goza de una vigencia extraordinaria el siglo XIII, pero a lo largo del otoño de la Edad Media y durante los Siglos de Oro esta forma aparece cada vez con menor frecuencia en la documentación.
} 
lengua escrita ${ }^{11}$ se observan en la mitad del siglo XVII. Aunque su empleo siempre ha sido bastante reducido, cabe suponer que la mayor productividad de esta forma tuvo lugar entre los siglos XIII y XVII.

2. En el corpus CORDE se recopilan contados ejemplos de la segunda persona del singular, de la primera persona del plural y de la segunda persona del plural del antefuturo de subjuntivo, lo cual confirma de algún modo que la forma en cuestión siempre ha sido típica del registro formal.

3. La forma bubiere cantado quedó reemplazada en primer lugar por haya cantado y cante, mientras que en la prótasis de oraciones condicionales, por restricciones puramente sintácticas, la sustituyeron las formas canta y ha cantado. Además, el contenido de no-realidad, que denotaba en algunos casos el antefuturo de subjuntivo, pasó a expresarse mediante el antecopretérito de subjuntivo (bubiera-se cantado).

4. El hecho de que bubiere cantado dejara de utilizarse no solo se debe a su contenido temporal y modal, sino también a numerosas restricciones sintácticas a las que se sometía dicha forma.

5. Sin hacer caso a la distinción entre los modos indicativo y subjuntivo, cabe destacar que bubiere cantado, al lado de hubo cantado, constituye la forma más débil del subsistema de tiempos compuestos que no consiguió resistir los cambios sucedidos en el sistema temporal castellano a partir de la Edad Media.

\footnotetext{
hablada

${ }^{11}$ Es perfectamente posible que esa tendencia se registrara más temprano en la lengua 


\section{BIBLIOGRAFÍA}

Álvarez Nazario, Manuel (1972), La herencia lingüistica de Canarias en Puerto Rico, San Juan: Instituto de Cultura Puertorriqueña.

Andres SuÁrez, Irene (1994), El verbo español. Sistemas medievales y sistema clásico, Madrid: Gredos.

Camús Bergareche, Bruno (1990), “El futuro de subjuntivo en español”, Ignacio Bosque (ed.), Indicativo y subjuntivo, Madrid: Taurus, pp. 410-427.

Commerelán y Gómez, Francisco A. (1881), Gramática de la lengua castellana compuesta con arreglo al plan y método más generalmente seguidos en la enseñanza del latín, y para facilitar a los alumnos de segunda enseñanza el estudio de este idioma, Madrid: Imprenta de A. Pérez Dubrull.

De Sterck, Goedele (2000), Registros y áreas geográficas en lingüistica. Usos y valores de las formas en -ra, -se, -ría y -re, Salamanca: Ediciones Universidad Salamanca.

Donni De Mirande, Nélida, E. (1984), "Sobre la variación diatópica del español en la Argentina", en Segundo Congreso Nacional de Lingüistica. Actas, I, San Juan: Universidad Nacional de San Juan, pp. 43-56.

Folgar, Carlos y Manuel Ramos (1992), "Privilegios de figuración del futuro de subjuntivo en español medieval”, Manuel Ariza Viguera et al. (eds.), Actas del II Congreso Internacional de Historia de la Lengua Española, Madrid: Pabellón de España, vol. I, pp. 403-427.

Gili Gaya, Samuel (1994 [1943]), Curso superior de sintaxis española, Barcelona: Bibliograf.

Granda, Germán de. (1968), "Formas en -re en español atlántico y problemas conexos”, BICC, XXIII, pp. 445-470.

Jorge Morel, Elercia (1974), Estudio lingüístico de Santo Domingo, Santo Domingo: Taller.

LENZ, Rodolfo (1944 [1920]), La oración y sus partes: Estudios de gramática general y castellana. Cuarta edición. Santiago de Chile: Editorial Nascimiento.

Lope Blanch, Juan M. (1972), Estudios sobre el español de México, Ciudad de México: UNAM.

Luquet, Gilles (1988), "Sobre la desaparición del futuro de subjuntivo en la lengua hablada de principios del siglo XVI", Manuel Ariza, Antonio Salvador y Antonio Viudas (eds.), Actas del I Congreso Internacional de Historia de la Lengua Española, Madrid: Arco/Libros, vol. I, pp. 509-514.

Moreno de Alba, José G. (2010 [1988]), El español en América, México: Fondo de Cultura Económica.

Nowikow, Wiaczesław (1993), Evolución funcional de los esquemas condicionales no reales en el español de los Siglos de Oro, Łódź/Frankfurt am Main: Wydawnictwo Uniwersytetu Łódzkiego/Vervuert.

Nowikow, Wiaczesław (1995), "Sobre las causas de la eliminación del futuro de subjuntivo del sistema verbal español”, Anna Bochnakowa y Stanisław Widłak (eds.), Munus Amicitiae. Studia Linguistica In Honorem Witoldi Mańczak Septuagenarii, Kraków: Uniwersytet Jagielloński, pp. 111-117.

Nowikow, Wiaczesław (2010), "En torno a la interferencia en la enseñanza de los pretéritos a los alumnos polacos: tiempo vs. Aspecto”, Gilles Luquet, Wiaczesław Nowikow, (eds.), La recherche en langues romanes. Théories et applications, Łódź: Lodz Academie of International Studies / Université Sorbonne Nouvelle-Paris 3, pp. 289-302.

Nowikow, Wiaczesław (2012), "La organización de los sistemas temporales del español y del polaco: análisis contrastivo de los tiempos verbales", Studia Iberystyczne, 11, pp. 119-134.

Octavio de Toledo y Huerta, Álvaro S. y Javier Rodríguez Molina (2008), "En busca del tiempo perdido: historia y uso de bube cantado”, Ángeles Carrasco Gutiérrez (ed.), Tiempos compuestos y formas verbales complejas, Madrid/Frankfurt: Iberoamericana/Vervuert, pp. 275-357.

PenNy, Ralph (2014), Gramática histórica del español, Barcelona: Ariel

Real Academia Española (2009), Nueva gramática de la lengua española, Madrid: Espasa Calpe. 
Rojo, Guillermo y Emilio Montero (1983), La evolución de los esquemas condicionales (Potenciales e irreales desde el Poema del Cid hasta 1400), Verba, 22.

SECO, Rafael (1990 [1930]), Manual de gramática española, Madrid: Aguilar.

VeIgA, Alexandre (1989), "La sustitución del futuro de subjuntivo en la diacronía del verbo español”, Verba, 16, pp. 257-338.

Veiga, Alexandre (2006), "Las formas verbales subjuntivas. Su reorganización modo-temporal”, Conepción Company Company (coord.), Sintaxis histórica de la lengua española (Primera parte, La frase verbal), vol. I, pp. 95-242.

Veiga, Alexandre (2011), "Algo más sobre la doble organización temporal en la diacronía del sistema verbal español”, Carsten Sinner, José L. Ramírez Luengo, María J. Torrens Álvarez (coords.), Tiempo, espacio y relaciones espacio-temporales desde la perspectiva de la lingüistica histórica, San Millán de la Cogolla: Cilengua, pp. 297-330.

Zamorano Aguilar, Alfonso (2005), El subjuntivo en la historia de la gramática española, 1771. 1973, Madrid: Arco/Libros, S.L.

\section{Corpus:}

Real Academia Española: Banco de datos (CORDE) Corpus diacrónico del español. http:// www.rae.es [Fecha de la consulta: 28.03-14.04.2020]

Recibido: agosto de 2020

Aceptado: diciembre de 2020 